\title{
EU/US/CTAD Task Force: Lessons Learned from Recent and Current Alzheimer's Prevention Trials
}

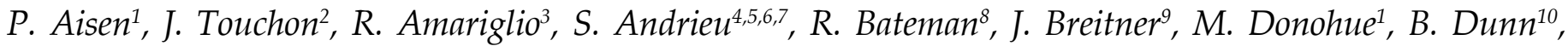 \\ R. Doody ${ }^{11}$, N. Fox ${ }^{12}$, S. Gauthier ${ }^{9}$, M. Grundman ${ }^{13}$, S. Hendrix ${ }^{14}$, C. Ho ${ }^{15}$, M. Isaac ${ }^{16}$, R. Raman ${ }^{1}$, \\ P. Rosenberg ${ }^{17}$, R. Schindler ${ }^{18}$, L. Schneider ${ }^{19}$, R. Sperling ${ }^{3}$, P. Tariot ${ }^{20}$, K. Welsh-Bohmer ${ }^{21}$, M. Weiner ${ }^{22}$, \\ B. Vellas ${ }^{4,5,6}$ and Task Force Members*
}

*E.U./U.S. CTAD TASK FORCE: Abushakra Susan (Framingham), Agus Sam (Valby), Aisen Paul (San Diego), Amariglio Rebecca (Boston), Andrieu Sandrine (Toulouse), Bateman Randall (St. Louis), Bowman Gene (Lausanne), Breitner John (Montreal), Budd Haeberlein Samantha (Cambridge), Cantillon Marc (Princeton), Carrillo Maria (Chicago), Clavier Isabelle (Chilly Mazarin), Cummings Jeffrey (Las Vegas), Dedieu Jean-François (Chilly Mazarin), Donohue Michael (San Diego), Doody Rachelle (Basel), Downing Ann Catherine (Indianapolis), Dubé Sanjay (Aliso Viejo), Dunn Billy (Chicago), Eggermont Laura (Utrecht), Fox Nick (London), Gauthier Serge (Verdun), Goedkoop René (Issy Les Moulineaux), Grundman Michael (San Diego), Hauge Vienna (Durham), Hendrix Suzanne (Salt Lake City), Ho Carole (South San Francisco), Krijezi Mubera (Basel), Lawson John (Malvern), Legrand Valérie (Nanterre), Malamut Rick (Aliso Viejo), McCarthy Marie (Leopardstown), Megerian Thomas (Aliso Viejo), Merdes Annette (Munich), Nosheny Rachel (San Francisco), Olsson Tina (Cambridge), Ondrus Matej (Bratislava), Pueyo Maria (Suresnes), Rafii Michael (San Diego), Raman Rema (San Diego), Raunig David (Dublin), Rosenberg Paul (Baltimore), Salloway Stephen (Providence), Schindler Rachel (New York), Shin Paul (Aliso Viejo), Siffert Joao (Cambridge), Sims John (Indianapolis), Sperling Reisa (Boston), Stephens Andrew (Berlin), Suhy Joyce (Newark), Tariot Pierre (Phoenix), Touchon Jacques (Montpellier), Tsukii Katsuyoshi (Princeton), Van Der Geyten Serge (Belgium), Vellas Bruno (Toulouse), Viglietta Vissia (Cambridge), Vincenzetti Christine (Lausanne), Weiner Michael (San Francisco), Welsh-Bohmer Kathleen (Durham), Wendland Jens (Cambridge), Wessels Alette (Indianapolis), Wilson Louisa (Princeton), Wunderlich Glen (Ridgefield), Yang Jerry (New York), Zimmer Jennifer (Indianapolis), Zoia Vanessa (Utrecht)

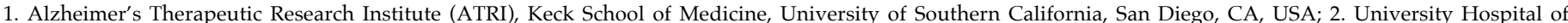

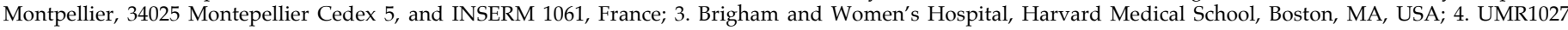

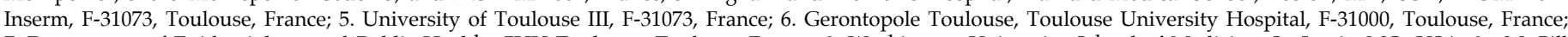

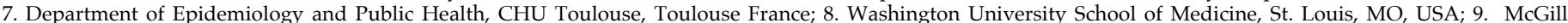

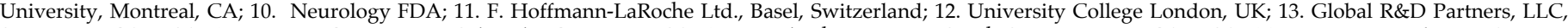

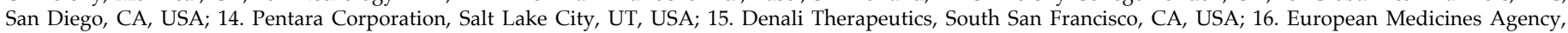

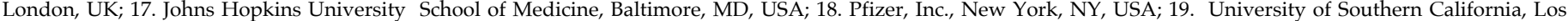
Angeles, CA USA; 20. Banner Alzheimer's Institute, Phoenix, AZ, USA; 21. Duke University, Durham, NC, USA; 22. University of California, San Francisco, CA, USA

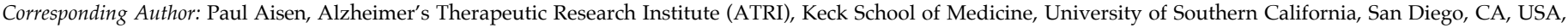
paisen@usc.edu

J Prev Alz Dis 2017;4(2):116-124

Published online April 25, 2017, http:/ / dx.doi.org/10.14283/jpad.2017.13

\begin{abstract}
At a meeting of the EU/US/Clinical Trials in Alzheimer's Disease (CTAD) Task Force in December 2016, an international group of investigators from industry, academia, and regulatory agencies reviewed lessons learned from ongoing and planned prevention trials, which will help guide future clinical trials of AD treatments, particularly in the pre-clinical space. The Task Force discussed challenges that need to be addressed across all aspects of clinical trials, calling for innovation in recruitment and retention, infrastructure development, and the selection of outcome measures. While cognitive change provides a marker of disease progression across the disease continuum, there remains a need to identify the optimal assessment tools that provide clinically meaningful endpoints. Patient- and informant-reported assessments of cognition and function may be useful but present additional challenges. Imaging and other biomarkers are also essential to maximize the efficiency of and the information learned from clinical trials.
\end{abstract}

Key words: Alzheimer's disease, clinical trials, secondary prevention trials, cognitive outcome measures, cognitive composites, patientreported outcome measures, informant-reported outcome measures, molecular imaging, mild behavioral impairment. 
clinical trial endpoints and promoting collaborations to improve the efficiency of clinical trials and promote data sharing $(1,2)$. Yet many challenges remain in the preclinical space, where our understanding of pathological mechanisms is still limited and where current tools may lack the sensitivity needed to optimize dosing regimens and detect clinically meaningful change.

\section{Prevention trials}

Prevention trials are underway across the spectrum of AD, from autosomal-dominant to sporadic, including populations with risk factors that increase the probability they will develop cognitive decline. Task Force participants described these complementary trials, emphasizing the cooperation, collaboration, and data sharing initiatives that have emerged.

\section{CAP - The Collaboration for Alzheimer's Prevention}

The Collaboration for Alzheimer's Prevention (CAP) is a partnership of the Alzheimer's Association, National Institute on Aging, Fidelity Foundation, US Food and Drug Administration (FDA), and four groups that are sponsoring five trials: the Alzheimer's Prevention Initiative (API), the Dominantly Inherited Alzheimer's Network Trials Unit (DIAN-TU), the Alzheimer's Therapeutic Research Institute (ATRI), and the TOMMORROW study. CAP brings these groups together under one umbrella to harmonize biomarkers, clinical, and cognitive measures, and align data- and samplesharing approaches used in these trials so that findings can be compared to inform the entire community (3).

\section{DIAN-TU}

While less than 1 percent of $\mathrm{AD}$ cases result from autosomal dominant mutations in three genes that are directly involved in $A \beta$ production, the predictable course of disease in these individuals provides an opportunity to model the disease, predict time of clinical onset, and intervene at any time point in the disease course due to the predictable time to biomarker changes and clinical symptom onset $(4,5)$. Findings from these autosomal dominant cases may also be translatable to sporadic populations (6).

DIAN, initiated as an observational study with the aim of characterizing the disease, has provided data in support of hypothetical models of disease progression (6, $7)$. This led to the idea that individuals could be targeted at various stages of disease. DIAN-TU has developed two trials:

The DIAN-TU-001 trial is a Phase $2 / 3$ placebocontrolled, double-blinded, cognitive outcome trial with biomarker interim analyses. Participants are mutation carriers or non-carriers (placebo controls only) between -15 to +10 years of estimated symptom onset with a global CDR of $0,0.5$, or 1 . Enrollment has been completed for this study. Mutation carriers were randomized to one of three arms: two different treatment arms (gantanerumab, solanezumab), or placebo in a 3:1 ratio of active to placebo. Drug treatment will continue for at least four years. The first two years are expected to enable establishment of a biomarker endpoint, and a cognitive endpoint will be compared after four years.

DIAN-TU will add two or more disease-modifying therapeutics to the platform in a trial called the Next Generation (NexGen) prevention trial, which will run in parallel and use an adaptive design (8). With a grant from the Alzheimer's Association, NexGen will add two new treatment arms, employ novel biomarkers, home-based cognitive testing, maximally effective dose adjustment, and may conduct a cognitive interim analysis. The disease-progression model used in the design of this study estimated decline based on observational cognitive data from presymptomatic participants in DIAN. DIAN-TU currently has trial performance sites in seven countries.

DIAN has already demonstrated that it is possible to predict clinical onset in those with ADAD mutations, allowing targeting of treatment to specific stages of disease. The DIAN-TU trial has highlighted other issues that are relevant to secondary prevention trials:

- Since potential participants do not have disease and may not have been involved in previous clinical trials, involving them in the design of the trial -- including decisions about enrollment and implementation of trial -- maximizes participant recruitment and retention.

- In addition to participants, family members, advocacy organizations, and pharmaceutical partners should be engaged in the development of the trial.

- Participant registries and cohorts developed from these registries are essential for efficient recruitment.

- Use of a defined population --such as those with ADAD mutations -- results in low rates of screen failures and thus can maximize the productivity of a trial.

- Attrition can be minimized by choosing expert trial sites with full commitment to the trial.

- Including biomarkers is essential to learn more about the effects of drugs.

\section{A4 and EARLY}

The Anti-Amyloid Treatment in Asymptomatic Alzheimer's (A4) study is a Phase 3 secondary prevention trial being conducted in partnership with Eli Lilly (9). It is enrolling clinically normal participants aged 65 to 85 thought to be at risk of developing cognitive decline due to AD based on evidence from an amyloid PET scan showing amyloid deposition in the brain. With support from Alzheimer's Association, A4 investigators will also 
follow a cohort of individuals with normal PET amyloid in the Longitudinal Evaluation of Amyloid Risk and Neurodegeneration (LEARN) Study; and with support from the National Institutes of Health's Accelerating Medicines Partnership (AMP), a subset of A4 participants will receive tau PET scans.

As of December 2016, enrollment for A4 has begun at 67 sites in the US, Canada, and Australia. More than 5,000 participants have been screened and 815 randomized. When enrollment is complete, 1150 participants will take part in the study. The trial is coordinated by the University of Southern California's ATRI.

A4 will utilize the Preclinical Alzheimer's Cognitive Composite (PACC) as the primary outcome measure (10). In parallel, the Harvard Aging Brain Study is evaluating a modification of the PACC that incorporates both the free and total scores of the free and cued selective reminding test (FCSRT) to see if they add power in early stage disease. Working with Janssen Pharmaceuticals, the ATRI team has also worked to initiate a global prevention study called EARLY in participants identified as amyloid positive by either PET scan or CSF analysis. This study will also include participants as young as age 60 with additional risk factors.

Lessons learned from A4 and EARLY include:

- Site start up and enrollment is challenging and has taken longer than was anticipated.

- Building infrastructure and trial-ready cohorts is essential to ensure that prevention studies can be completed in a reasonable time frame.

\section{API}

The Alzheimer's Prevention Initiative, established by the Banner Alzheimer's Institute in Phoenix, Arizona, to evaluate disease-modifying treatments for Alzheimer's disease (11), has launched two trials in cognitively unimpaired people who are at high imminent risk at the time of enrollment. The first of these trials -- the API-ADAD trial (NCT01998841)- enrolled individuals from large kindreds in Antioquia, Colombia, with the autosomal dominant PSEN1 E280A mutation, which virtually ensures that carriers will develop early onset AD. Both mutation carriers and non-carriers are enrolled, although their mutation status is not disclosed through an interesting design that embeds two substudies: 1) a randomized clinical trial in which only mutation carriers are randomized to receive either placebo or treatment; and 2) a cohort study that compares mutation carriers and non-carriers receiving placebo. The 60-month study was launched in 2013.

Lessons learned from API include:

- The important enabling role of Health Authorities

- The importance of existing and new data, including biomarker data, upon which to base the design

- The value of a registry for recruitment, which allowed balancing of carriers and non-carriers referred to the study while maintaining blind to genotype

- Pre-screen fail rates were high because of prohibited medical conditions, mild cognitive impairment, illiteracy, low MMSE, and scheduling.

- Screen fail rates were also higher than predicted because of labs, medical conditions, inability to comply with the protocol, and MMSE.

- Participants were exceptionally motivated and the team implemented well-planned adherence and retention strategies that resulted in only $2.6 \%$ drop out rate compared to $25 \%$ predicted. This has helped to preserve the power of the study.

- Collaboration with colleagues at the Grupo Neurosciences de Antioquia (GNA) was essential to address substantial cultural, ethnic, and language issues. This included setting up a "health plan" in Colombia to assure access to health care and a "social plan" to support families regardless of whether they were participating in the study.

- Flexibility was required in terms of adapting the trial to new findings (e.g., increasing the doses of crenezumab and embedding tau PET), adapting to changes in the sponsor team over time, the continuing need for funding, and responding to media attention.

Unanticipated issues that arose included low vitamin B12 levels, low thyroid function tests, and a high prevalence of people with limited formal education that produced challenges in obtaining proper informed consent. To accommodate those with low literacy, the study team created an informed consent form in the form of an illustrated companion guide.

Moving forward, the API-ADAD team may need to address a variety of issues, including responding to changes in community standards regarding genetic testing and disclosure, determining when and how to implement new studies, extending the trial in a way that maximizes power and retention but minimizes disclosure of genetic status, following participants after the conclusion of the study, establishing the clinical meaningfulness of differences in cognition, and introducing the possibility of autopsy studies.

The second API study - the Generation Study - will enroll about 1340 participants between the ages of 60 and 75 who are cognitively unimpaired and homozygous for the ApoEc4 allele, which dramatically increases an individual's risk of developing late onset AD.

\section{MAPT}

The Multi-domain Alzheimer's Prevention Trial (MAPT) is a Phase 3 randomized, placebo-controlled intervention study conducted at multiple sites across France, which tested a multi-domain intervention comprising nutrition, physical exercise, cognitive and social activities, and an increased intake of omega-3 polyunsaturated fatty acid in frail older adults at risk of 
cognitive decline (12). 1680 adults age 70 or older with subjective memory complaints but no dementia and living in community settings were enrolled in the 5-year study that included 3 years of intervention plus 2 years of additional observation. The primary outcome measure was cognitive decline, assessed using an adapted version of the PACC. Subgroups of participants also had imaging studies. Preliminary results suggest that the multidomain intervention slowed cognitive decline compared to the placebo group, although the primary outcome was not significant after adjusting for multiple comparisons. Placebo group data also demonstrated increased cognitive decline in participants who were amyloidpositive, ApoE 44 carriers, those who had a baseline CDR of 0.5 suggesting mild cognitive impairment (MCI), older individuals ( $+75 \mathrm{yrs})$, and those with lower blood levels of Docosahexaenoic acid (DHA) and Eicosapentaenoic acid (EPA) (13).

Lessons learned from MAPT include:

- Excluding participants who are less likely to decline can increase the ability of the trial to detect an effect.

- Including participants with early MCI can increase the power of the trial because they are more likely to decline without intervention.

- Cognitive composites are useful; however learning effects are important and need to be controlled for. Practice sessions before randomization are recommended.

- Local and regional networks of research centers, memory clinics, and family practitioners are essential for recruitment. Mobile research teams may augment these sites.

- Home-based visits may limit the number of dropouts.

Further trials are currently in development to follow up on the MAPT results:

- LowMapt is a randomized placebo control study targeting those with low DHA in red blood cells. The objective is to replicate the cognitive effect observed in MAPT subjects with low DHA/EPA. Target population: Older adults 70 yrs old,$+ N=400$, with low DHA/EPA RBC < 4.83\%; Intervention: DHA 800 mg/EPA $500 \mathrm{mg}$ vs placebo. Duration: 18 months, plus supplementation for 18 months, total 36 months. Delayed start analysis. Primary Criteria: Cognitive Composite score

- Nolan trail: The objective is to prevent cognitive decline in older adults with memory complaint with a Brain Protector Blend (Nestle Research center) versus placebo. Target population: 2080 subjects, +70 yrs with Memory complaints but no dementia. 4 years of follow-up. Co-primary subgroup sizes: Low DHA/ EPA subgroup: $n=646$ CDR 0.5 subgroup: $n=580$. Primary criteria: MAPT Cognitive Composite Score.

- MAPT - e-Study. The objective is to replicate the multi-domain intervention observed effect using new technologies. Target population: Older adults $70+\mathrm{yrs}$ old with memory complaint, $\mathrm{N}=120$. R.C.T: e-Multidomain intervention, using e-platform, and e-coach vs usual follow up. Duration: 6 months. Primary Criteria: cognitive composite score, both paper and electronic.

\section{TOMMORROW}

The TOMMORROW study is a multi-national, randomized, placebo-controlled trial designed to simultaneously test two co-primary hypotheses. The first is whether low-dose pioglitazone, which modulates the transcription of genes involved in glucose and lipid metabolism, can delay the onset of MCI due to AD in a population enriched for those for carriers of the TOMM40 rs10524523 gene and the ApoEc4 allele, which increases their risk of cognitive decline. The second aim is to assess the predictive utility of a genetic biomarker algorithm comprised of age, APOE and TOMM40 geneotypes in the near-term onset of symptoms due to MCI due to AD. The study uses a time to event design for both aims. The primary endpoint is a clinical diagnosis of MCI due to AD which uses operationalized criteria that have been cross-culturally validated allowing for harmonized diagnostic assignment across the nearly 60 sites involved in the global trial.

Lessons learned from TOMMORROW include:

- Use of a streamlined battery of neuropsychological tests, akin to clinical practice, tapping domains of verbal and visual memory, language, visuospatial function, executive control, and attention was intended to capture the heterogeneity in early MCI due to $\mathrm{AD}$ and may provide new insights into the earliest cognitive manifestations of emerging $\mathrm{MCI}$ due to $\mathrm{AD}$

- Variability in cognitive measurement can be a limitation in clinical trials that rest on these endpoints. The TOMMORROW study with its diagnostic endpoint requires clinical neuropsychologists at each site and provides tight external quality assurance provided through study vendors. The latter ensures standardized administration of all measures across sites, and provides centralized scoring of measures which are inherently more variable in their scoring (e.g visuoconstruction and visual memory measures)

- A clinical diagnosis of MCI due to AD based on the clinical criteria of the 2011 NIA-Alzheimer's Association (14) is a novel endpoint in trials. This diagnosis has been rigorously operationalized for global use and is defined as

o a decline from a baseline CDR score of 0 to a score of 0.5 and

o failure either on one of two memory tests (-1.5 SD below an age adjusted mean and a change from baseline) or failure in 2 of 12 measures in separate domains of which one is memory (-1.3 SD below normative mean and a change from baseline)

o exclusion of competing medical explanations 
- And importantly, to be a confirmed MCI-AD endpoint the clinical diagnosis must be confirmed across two consecutive observations 6 months apart. And all primary endpoint events are affirmed by an independent, blinded adjudication panel, allowing harmonization in the diagnosis across clinicians, languages, and cultures.

- Selecting sites on the basis of access to a large population of healthy elderly, the availability of site registries, and dedicated staff able to manage a high number of participant visits can maximize the success of enrollment.

- Developing a customized recruitment strategy for each site may be needed.

- Validating instruments and establishing normative cutpoints in different languages and communities is needed for multi-national studies.

\section{Outcome measures for prevention trials}

Cognitive change is an early change that can be detected in preclinical AD and is a manifestation of $\mathrm{AD}$, making it possibly the best "biomarker" for $\mathrm{AD}$ trials, including preclinical trials. Assessing cognition represents a unifying approach to measurement of disease progression and can be adapted as an outcome measure for clinical trials, since it has face validity and directional hypotheses can be postulated a priori. However, there remain concerns about the clinical meaningfulness of some cognitive measures since points on scales do not always correspond to a clinically meaningful benefit. The sensitivity of individual cognitive measures has also been called into question in the earliest stages of disease. Regulatory agencies including the FDA and the European Medicines Agency (EMA) have issued draft guidance on developing treatments for early stage disease that require endpoints to include functional and global measures in addition to cognition $(15,16)$, and multiple analyses have concluded that composites incorporating both cognitive and functional measures may increase power in a trial in preclinical AD $(17,18)$. Clinical endpoints, based on a diagnosis of dementia or MCI have also been used in some trials, including the TOMMORROW trial.

\section{Cognitive composites}

Several different cognitive batteries and composites have been created for the prevention trials described above. There are significant similarities among these composites in terms of domains and constructs, although they may use different instruments to assess episodic memory, executive function, orientation, and other domains. Some of the composites include semantic measures like category fluency. Composite measures, and the weights assigned to different components, may be theoretically or empirically driven, or may have elements of both approaches. They can be optimized for clinical progression or for different stages of disease. The similarity among these composites supports the notion that cognition is a special marker in the AD field and that it is useful across the entire spectrum of disease including the preclinical stage. Whether such composites are useful in primary prevention studies remains to be determined.

The PACC includes, in addition to cognitive measures across multiple domains, the mini-mental status exam (MMSE) to assess global functioning and mental status. An analysis of scores from individuals in the AIBL study with elevated $A \beta$ suggested that dropping the MMSE improves sensitivity in the preclinical stage of disease (19). However, studies in other populations, including DIAN, ADNI, API, and PAQUID, indicate that MMSE scores separate 6-9 years before dementia diagnosis even in people with elevated $A \beta(6,20-22)$. The APCC includes only the orientation to time from the MMSE, based on data from three combined studies: Rush Alzheimer's Disease Center's Religious Orders Study [ROS], Memory and Aging Project [MAP], and the Minority Aging Research Study [MARS], which indicated that other MMSE items did not improve sensitivity to progression in preclinical stages (23). These different conclusions, and in particular a study conducted by Donohue and colleagues (21), suggest that cross-validation should be conducted when considering changes to composite measures.

There may be additional cognitive components that are not captured by current composites, such as differentiating between processing speed, difficulty with a task, and the ability to learn new words. In addition, cognitive composites fail to capture declines in social functioning such as participating in conversations and navigating social situations.

Cognitive composites and online tools for assessing cognition may also be useful to gather data in general populations as a screening tool.

\section{Computerized cognitive assessments}

Computerized cognitive assessments and computerized cognitive batteries have been suggested as providing more reliable and efficient means of assessing cognition compared to paper and pencil measures. In a pilot study of clinically normal older adults comparing two computerized batteries -- the NIH Toolbox Cognition Battery (NIHTB-CB) and the Cogstate iPad C3 battery - to the PACC, both computerized batteries showed promise. The Cogstate-C3 provides two distinct composites; one measuring logical memory and the other measuring processing speed and attention. Both the NIHTB-CB and the $\mathrm{C} 3$ Learning-Memory composites correlated well with the PACC, and the C3 Learning-Memory composite also identified subtle cognitive impairment with the greatest sensitivity and specificity. The NIHTB-CB showed the strongest overall clustering and alignment with the 
PACC. The authors concluded that further testing will be needed before these measures can be used in large scale prevention trials (24).

\section{Patient and informant-reported outcomes}

Given the need for outcome measures that are clinically meaningful, other options that have been considered include performance-based functional measures and informant- or patient-reported activities of daily living (ADL) or instrumental activities of daily living (iADL) scales. Performance-based functional measures include assessments of financial capacity (25), ability to perform an automated phone task $(26,27)$, and a virtual reality simulation of functional abilities related to shopping (taking a bus, shopping, managing money) (28). The latter was developed for schizophrenia, not dementia trials. Patient- and informant-based scales include the ADCS-ADL scale (29), the Everyday Cognition (E-Cog) scale (30), the Cognitive Function Instrument (31), the Functional Activities Questionnaire (FAQ) (32), and the Amsterdam IADL Questionnaire (33). A major advantage of performance-based measures is that they capture changes in everyday function, which reflect clinically meaningful deterioration. Patient- and informant-rated outcomes (PROs and IROs) may be easier to administer and can cover a broad range of everyday tasks that include both cognitive (e.g., repeating oneself) and functional changes (e.g., difficulty with driving). Initially, individuals may notice changes that are imperceptible to others, making them especially useful in early disease stages. However, as the disease progresses, patients may lose awareness of their impairments, making IROs potentially more useful, although the point along the trajectory where this happens is unclear and variable. Switching from PROs to IROs as disease progresses in a clinical trial could be particularly challenging.

Self-reported measures of subjective cognitive decline have also been proposed by an international working group (34). A review of self-report measures used in 19 international research studies reported wide heterogeneity across measures (35). To develop a more reliable subjective cognitive decline measure, the working group recommended asking specific rather than broad questions, with specific time references (e.g., change from one year ago), and including questions about mood, personality, and health factors. However, subjective cognitive decline measures are sensitive to various biases. In recent analyses by the Harvard Aging Brain Study, the relationship between subjective cognitive decline and cognition was shown to be stronger among Caucasians than African Americans; and the relationship between subjective report and amyloid burden was shown to be stronger in those with more education compared to those with less education (36).

\section{Imaging}

Imaging provides structural, molecular, and functional information about AD that can help guide decisions about potential clinical benefits of treatments and provide information on mechanisms and safety. These measures can thus be used either as inclusion criteria or outcomes. Most of the prevention studies discussed above incorporate structural MRI as well as amyloid and tau PET. DIAN-TU and API-ADAD also include fluorodeoxyglucose (FDG) PET, which measures brain metabolism; and A4 and DIAN-TU add task-free functional MRI studies to assess the functioning of neural networks.

Selection of imaging endpoints as outcome measures in trials depends on the treatment mechanism (e.g., targeting amyloid, tau, neuroinflammation, or neurodegeneration); the aim of the study (e.g., primary, secondary, or tertiary prevention); the desired outcome (e.g., slowing, stopping, or reversing accumulation of tau or amyloid or neurodegeneration); and subject selection (i.e., pathology and stage of disease). A single imaging marker such as amyloid deposition may show the presence of disease, but the long period (10-15 years) when preclinical individuals may have evidence of cerebral amyloid means that using amyloid as the sole inclusion measure can lead to substantial heterogeneity, thus reducing statistical power (37). This can be mitigated by combining imaging and other biomarkers (38). Combining biomarkers may provide a better understanding of the effects of therapy.

A wide range of new imaging markers of molecular pathology and neurodegeneration are becoming available, such as PET ligands that enable assessment of neuroinflammation and synaptic density. These new markers may enable trials targeted more specifically to certain types of treatment and stages of disease, but will require the field to share data and align on standardized methods and develop an evidence base demonstrating optimal sample sizes to predict potentially clinically meaningful benefit.

\section{Early behavioral disorders in preclinical $A D$}

Early neuropsychiatric and behavioral symptoms and disorders may also be useful indicators of preclinical $\mathrm{AD}$, a concept that has been termed "Mild Behavioral Impairment" (MBI), akin to MCI and with recent publication of provisional criteria and a checklist (39, 40). Multiple studies have demonstrated an association between neuropsychiatric symptoms and an increased risk of dementia and $\mathrm{AD}$ (41-44), although the mechanisms underlying this association remain unclear. While some studies have suggested that depression is associated with an increase in accumulation of brain amyloid, neurofibrillary tangles, or hippocampal atrophy (45-47), others have shown no association between 
dementia-related markers of pathology and depression (48). Anxiety has also been linked to increased levels of plaques and tangles (46). Self-reported loneliness has also been associated with elevated brain amyloid (49), suggesting that measurement of loneliness and other neuropsychiatric symptoms not captured by currently used measures such as the Neuropsychiatric Inventory (NPI), Geriatric Depression Scale, or Patient Health Questionnaire-9 (50) may add to the armamentarium of tools to detect preclinical AD.

\section{Regulatory considerations}

While cognition is unquestionably important, regulators continue to express concerns about the assessment tools currently available and their ability to identify clinically meaningful change in the early stages of AD. Likewise, there is a need for more sensitive measures of functional impact that reflect cognitive domains disturbed in early disease. Bridging the space between cognition and function is critical, leading to an increased reliance on performance-based and patientreported outcomes. However, demonstrating the reliability of data collected using these measures remains a challenge. Moreover, given that clinical meaningfulness may change across the continuum of the disease, outcome measures used in clinical trials may also need to change depending on the stage of disease. Safety is another important criterion for regulators; however, risktolerance and the risk/benefit tradeoff may also change as the disease progresses, adding further complexity to regulatory decisions. This requires the inclusions of patients and patient representatives in the decisionmaking process.

\section{Conclusions}

There are reasons for optimism regarding drug development for $\mathrm{AD}$, including an improved understanding of the biological mechanisms underlying early stage disease, more data sharing and collaboration, new assessment tools and biomarkers (e.g. tau PET, remote cognitive assessments), and the establishment of several different registries of potential trial participants. Approval and acceptance of a central IRB mechanism, which should markedly improve trial enrollment, is expected in 2017.

However, many challenges remain. Trials continue to take too long and cost too much. Phase 2 studies continue to be poor at predicting success in Phase 3. Tackling the problem of high screen failure rates, resulting from exclusions for co-morbidities or the presence or absence of genetic factors or mild cognitive impairment, will be essential to enable the enrollment of study populations that reflect the real-world population that preventive interventions are designed for. Including individuals with diabetes, cerebrovascular disease, and other risk factors will be necessary, but will require complex multivariate analyses.

Biomarker disappointments suggest a lack of shortcuts to demonstrating efficacy as well as the need for further standardization. Validating biomarkers is a key necessary step to facilitate future studies. While PET imaging has been incorporated into many prevention trials, CSF studies offer a potentially less expensive alternative to assess amyloid or tau load. In many countries outside of the US, lumbar puncture has a higher degree of acceptability among both patients and practitioners. However, since CSF and PET studies provide different information about pharmacodynamics and accumulation of amyloid, they are not completely interchangeable. There is also a need for more sensitive biomarkers that are pathway-independent but could assess cognitive loss, such as markers of synaptic function, axon degeneration, neuroinflammation. Alignment of the research community around some lead candidates to incorporate into studies could accelerate the identification and development of these novel biomarkers.

Since no one drug is likely to work across all population groups, tools need to be adapted to assess change in trajectory across different disease stages and population groups. In designing a trial and selecting the most appropriate assessment tools, trialists should keep in mind that the best trial may be the simplest trial, since burden on participants, families, sites, and operational teams can sink an otherwise excellent trial design.

Improved infrastructure is needed. Building a network of trial sites that use simplified contract language, a common, standardized set of methods, have pre-trained raters and other personnel, and have contracts in place so they can start trials quickly could reduce lengthy start-up times and optimize data management. As part of a new paradigm for Alzheimer's prevention, one suggestion was to establish Alzheimer's prevention programs that are independent of hospital-based memory centers and more focused on health promotion. Another suggestion was to create trial-ready populations within existing health care systems, despite the challenges of conducting studies in clinical care settings, including issues related to reimbursement when services are provided in the context of a clinical trial. Trial-ready organizations that can be quickly responsive are clearly needed. The GAPNet program has infused $\$ 100,000$ into 11 sites to try to develop a science of recruitment; 43 additional sites will be activated this year, 60 percent of these academic and 40 percent commercial. A central IRB is also crucial, and $\mathrm{NIH}$ has helped by requiring it for multi-site NIH studies. In the US, the NIA recently announced a $\$ 70$ million fiveyear award to establish an Alzheimer's Clinical Trials Consortium (ACTC) that will include multiple trial sites. Cooperation between ACTC, the European Prevention of Alzheimer's Disease (EPAD) consortium, and other studies will be needed to ensure synergy with European efforts. 
Resources are also needed for outreach and recruitment, recognizing that different approaches may be appropriate for different populations. One example of progress in this area was reported by the Arizona Alzheimer's Consortium, which created a registry with multiple goals: increasing awareness of $\mathrm{AD}$ research and prescreening, screening and referring eager registrants to studies (51). API has also created a registry, the Alzheimer's Prevention Registry, which has demonstrated recruitment success at local events. National branding efforts can also be useful if they match what is being done at the local level. Working with the media can also boost recruitment, but sites must be prepared to respond quickly to possibly hundreds of calls when a major story comes out in the news. Registries also offer opportunities to collect pre-randomization cognitive or functional data that can help document disease trajectories in cohorts before they develop disease.

Thinking of $\mathrm{AD}$ as a single disease occurring across a continuum also can provide a regulatory benefit by allowing trials to combine participants at different stages of disease. In addition, both the FDA and EMA have created mechanisms that allow the approval or conditional approval of a drug if it is "reasonably likely" that a positive signal on a cognitive measure or biomarker will translate into a clinically meaningful benefit even in the absence of two confirmatory pivotal trials establishing efficacy. These mechanisms typically require post-approval studies showing functional benefits, which may also provide data for payers about the real-world benefits of a treatment. Indeed, since the ultimate goal of drug development is to move a drug to market so it can provide benefits to patients, attention to payer considerations is needed throughout the drug development process. In this regard, considering cost savings as an outcome measure may provide data important to payers.

Finally, the EU US CTAD Task Force recommended investing in the next generation to train and encourage them to become clinicians, neuropsychologists, quantitative researchers, and clinical trialists, since these professionals will be essential for sustaining longduration prevention studies and continuing progress toward effective treatments and cures.

Acknowledgements: The authors thank Lisa J. Bain for assistance in the preparation of this manuscript.

Conflict of interest: P. Aisen: NeuroPhage, Eli Lilly, Merck, Roche, Amgen, Abbvie, Pfizer, Novartis, Janssen, Lundbeck, Biogen, iPerian, Probiodrug, Anavex, Cohbar, Cytox, aTyr, Avanir; R. Bateman: Abbvie, AstraZeneca, Biogen, Eisai, Eli Lilly and Co., Hoffman La-Roche Inc., Janssen, Pfizer, Sanofi-Aventi; N. Fox: Janssen, Roche, Eli Lilly, Novartis, GSK, Biogen; S. Gauthier: Eli Lilly, TauRx, Roche, Eisai, Lundbeck, Schwabe; L. Schneider: Eli Lilly, Novartis, Roche/Genentech, Takeda; P. Tariot: Roche, Novartis, Amgen, Abbvie, Eli Lilly, AstraZeneca, Lundbeck, AC Immune, Merck, Takeda, Boehringer-Ingelheim; K. Welsh-Bohmer: Takeda, Merck, Biogen, Roche, J\&J; B. Vellas: Biogen, Eli Lilly, Roche, Nestlé, Merck; R. Sperling: Avid/Lilly, Janssen, Bracket, Genetech, Sanofi, Roche, Abbvie, Lundbeck, Otsuka, Merck. S. Henrix: Pentara Corporation; R. Doody: Genentech/Roche; C. Ho, J. Breitner, R. Amariglio, M. Grundman, R. Raman, M. Isaac: No conflict of interest. The Task Force was partially funded by registration fees from industrial participants. These corporations placed no restrictions on this work.

\section{References}

1. Aisen P, Touchon J, Andrieu S, Boada M, Doody R, Nosheny R, et al. Registries and cohorts to accelerate early phase Alzheimer's trials. A report from the EU/US Clinical Trials in Alzheimer's Disease Task Force. J Prev Alz Dis. 2016;3(2):68-74.

2. Vellas B, Bateman R, Blennow K, Frisoni G, Johnson K, Katz R, et al. Endpoints for Pre-Dementia AD Trials: A Report from the EU/US/CTAD Task Force. J Prev Alzheimers Dis. 2015;2(2):128-35.

3. Reiman EM, Langbaum JB, Tariot PN, Lopera F, Bateman RJ, Morris JC, et al. CAP--advancing the evaluation of preclinical Alzheimer disease treatments. Nat Rev Neurol. 2016;12(1):56-61.

4. Ryman DC, Acosta-Baena N, Aisen PS, Bird T, Danek A, Fox NC, et al. Symptom onset in autosomal dominant Alzheimer disease: a systematic review and meta-analysis. Neurology. 2014;83(3):253-60.

5. Ryan NS, Nicholas JM, Weston PS, Liang Y, Lashley T, Guerreiro R, et al Clinical phenotype and genetic associations in autosomal dominant familial Alzheimer's disease: a case series. Lancet Neurol. 2016;15(13):1326-35.

6. Bateman RJ, Xiong C, Benzinger TL, Fagan AM, Goate A, Fox NC, et al Clinical and biomarker changes in dominantly inherited Alzheimer's disease. N Engl J Med. 2012;367(9):795-804.

7. Jack CR, Jr., Knopman DS, Jagust WJ, Shaw LM, Aisen PS, Weiner MW, et al Hypothetical model of dynamic biomarkers of the Alzheimer's pathological cascade. Lancet Neurol. 2010;9(1):119-28.

8. Bateman RJ, Benzinger TL, Berry S, Clifford DB, Duggan C, Fagan AM, et al. The DIAN-TU Next Generation Alzheimer's prevention trial: Adaptive design and disease progression model. Alzheimers Dement. 2017;13(1):8-19.

9. Sperling RA, Rentz DM, Johnson KA, Karlawish J, Donohue M, Salmon DP et al. The A4 Study: Stopping AD Before Symptoms Begin? Sci Transl Med. 2014;6(228):228fs13.

10. Donohue MC, Sperling RA, Salmon DP, Rentz DM, Raman R, Thomas RG, et al. The preclinical Alzheimer cognitive composite: measuring amyloidrelated decline. JAMA Neurol. 2014;71(8):961-70.

11. Reiman EM, Langbaum JB, Fleisher AS, Caselli RJ, Chen K, Ayutyanont N, et al. Alzheimer's Prevention Initiative: a plan to accelerate the evaluation of presymptomatic treatments. J Alzheimers Dis. 2011;26 Suppl 3:321-9.

12. Vellas B, Carrie I, Gillette-Guyonnet S, Touchon J, Dantoine T, Dartigues JF, et al. Mapt Study: A Multidomain Approach for Preventing Alzheimer's Disease: Design and Baseline Data. J Prev Alzheimers Dis. 2014;1(1):13-22.

13. Weiser MJ, Butt CM, Mohajeri MH. Docosahexaenoic Acid and Cognition throughout the Lifespan. Nutrients. 2016;8(2):99.

14. Albert MS, Dekosky ST, Dickson D, Dubois B, Feldman HH, Fox NC, et al. The diagnosis of mild cognitive impairment due to Alzheimer's disease: Recommendations from the National Institute on Aging-Alzheimer's Association workgroups on diagnostic guidelines for Alzheimer's disease. Alzheimers Dement. 2011;7(3):270-9.

15. European Medicines Agency. Draft guideline on the clinical investigation of medicines for the treatment of Alzheimer's disease and other dementia. In (CHMP) CfMPfHU, editor. London, United KIngdom2016.

16. Food and Drug Administration. Draft Guidance for Industry. Alzheimer's disease: Developing drugs for the treatment of early stage disease. Accessed 2-24-2013 at http://www.fda.gov/downloads/Drugs/ GuidanceComplianceRegulatoryInformation/Guidances / UCM338287.pdf; 2013.

17. Insel PS, Donohue MC, Mackin RS, Aisen PS, Hansson O, Weiner MW, et al. Cognitive and functional changes associated with Abeta pathology and the progression to mild cognitive impairment. Neurobiol Aging. 2016;48:172-81.

18. Wang J, Logovinsky V, Hendrix SB, Stanworth SH, Perdomo C, Xu L, et al. ADCOMS: a composite clinical outcome for prodromal Alzheimer's disease trials. J Neurol Neurosurg Psychiatry. 2016;87(9):993-9.

19. Lim YY, Snyder PJ, Pietrzak RH, Ukiqi A, Villemagne VL, Ames D, et al. Sensitivity of composite scores to amyloid burden in preclinical Alzheimer's disease: Introducing the Z-scores of Attention, Verbal fluency, and Episodic memory for Nondemented older adults composite score. Alzheimers Dement (Amst). 2016;2:19-26.

20. Amieva H, Le Goff M, Millet X, Orgogozo JM, Peres K, Barberger-Gateau P, et al. Prodromal Alzheimer's disease: successive emergence of the clinical symptoms. Ann Neurol. 2008;64(5):492-8.

21. Donohue MC, Sun C-K, Raman R, Insel PS, Aisen P. Cross-validation of optimized composites for preclinical Alzheimer. Alzheimers Dement TRCI 2017;in press.

22. Fleisher A, Chen K, Quiroz Y, et al. Associations between biomarkers and age in the presenilin $1 \mathrm{E} 280 \mathrm{~A}$ autosomal dominant Alzheimer's disease kindred: a cross-sectional study JAMA Neurology. 2015;in press

23. Langbaum JB, Hendrix SB, Ayutyanont N, Chen K, Fleisher AS, Shah RC, et al. An empirically derived composite cognitive test score with improved power to track and evaluate treatments for preclinical Alzheimer's disease. Alzheimers Dement. 2014;10(6):666-74.

24. Buckley RF, Sparks KP, Papp KV, Dekhtyar M, Martin C, Burnham S, et al. Computerized cognitive testing for use in clinical trials: A comparison of the 
NIH Toolbox and Cogstate C3 batteries. J Prev Alz Dis. 2017;4(1):3-11.

25. Marson DC, Sawrie SM, Snyder S, McInturff B, Stalvey T, Boothe A, et al. Assessing financial capacity in patients with Alzheimer disease: A conceptual model and prototype instrument. Arch Neurol. 2000;57(6):877-84.

26. Marshall GA, Dekhtyar M, Bruno JM, Jethwani K, Amariglio RE, Johnson KA, et al. The Harvard Automated Phone Task: new performance-based activities of daily living tests for early Alzheimer's disease. J Prev Alzheimers Dis. 2015;2(4):242-53.

27. Marshall GA, Aghjayan SL, Dekhtyar M, Locascio JJ, Jethwani K, Amariglio $\mathrm{R}$, et al. Activities of daily living measured by the Harvard Automated Phote Task track with cognitive decline over time in non-demented elderly. J Prev Alz Dis. 2017;in press.

28. Keefe RS, Davis VG, Atkins AS, Vaughan A, Patterson T, Narasimhan M, et al. Validation of a Computerized test of Functional Capacity. Schizophr Res. 2016;175(1-3):90-6.

29. Galasko D, Bennett DA, Sano M, Marson D, Kaye J, Edland SD, et al. ADCS Prevention Instrument Project: assessment of instrumental activities of daily living for community-dwelling elderly individuals in dementia prevention clinical trials. Alzheimer Dis Assoc Disord. 2006;20(4 Suppl 3):S152-69.

30. Farias ST, Mungas D, Reed BR, Cahn-Weiner D, Jagust W, Baynes K, et al. The measurement of everyday cognition (ECog): scale development and psychometric properties. Neuropsychology. 2008;22(4):531-44.

31. Amariglio RE, Donohue MC, Marshall GA, Rentz DM, Salmon DP, Ferris SH, et al. Tracking early decline in cognitive function in older individuals at risk for Alzheimer disease dementia: the Alzheimer's Disease Cooperative Study Cognitive Function Instrument. JAMA Neurol. 2015;72(4):446-54.

32. Pfeiffer RI, Kurosaki TT, Harrah CH, Jr., Chance JM, Filos S. Measurement of functional abilities in older adults in the community. J Gerontol. 1982;37(3):323-9.

33. Sikkes SA, de Lange-de Klerk ES, Pijnenburg YA, Gillissen F, Romkes R, Knol $\mathrm{DL}$, et al. A new informant-based questionnaire for instrumental activities of daily living in dementia. Alzheimers Dement. 2012;8(6):536-43.

34. Jessen F, Amariglio RE, van Boxtel M, Breteler M, Ceccaldi M, Chetelat G, et al. A conceptual framework for research on subjective cognitive decline in preclinical Alzheimer's disease. Alzheimers Dement. 2014;10(6):844-52.

35. Rabin LA, Smart CM, Crane PK, Amariglio RE, Berman LM, Boada M, et al. Subjective Cognitive Decline in Older Adults: An Overview of Self-Report Measures Used Across 19 International Research Studies. J Alzheimers Dis. 2015;48 Suppl 1:S63-86.

36. Aghjayan SL, Buckley RF, Vannini P, Rentz DM, Jackson JD, Sperling RA, et al. The influence of demographic factors on subjective cognitive concerns and beta-amyloid. Int Psychogeriatr. 2016:1-8.

37. Villemagne VL, Burnham S, Bourgeat P, Brown B, Ellis KA, Salvado O, et al. Amyloid beta deposition, neurodegeneration, and cognitive decline in sporadic Alzheimer's disease: a prospective cohort study. Lancet Neurol. 2013;12(4):357-67.

38. Salloway S, Sperling R, Fox NC, Blennow K, Klunk W, Raskind M, et al. Two phase 3 trials of bapineuzumab in mild-to-moderate Alzheimer's disease. $\mathrm{N}$
Engl J Med. 2014;370(4):322-33.

39. Ismail Z, Aguera-Ortiz L, Brodaty H, Ciestak A, Cummings J, Geda YE, et al. The Mild Behavioral Impairment Checklist (MBI-C): A rating scale for neuropsychiatric symptoms in pre-dementia populations. J Alzheimers Dis. 2017;56(3):929-38

40. Ismail Z, Smith EE, Geda Y, Sultzer D, Brodaty H, Smith G, et al. Neuropsychiatric symptoms as early manifestations of emergent dementia: Provisional diagnostic criteria for mild behavioral impairment. Alzheimers Dement. 2016;12(2):195-202.

41. Banks SJ, Raman R, He F, Salmon DP, Ferris S, Aisen P, et al. The Alzheimer's disease cooperative study prevention instrument project: longitudinal outcome of behavioral measures as predictors of cognitive decline. Dement Geriatr Cogn Dis Extra. 2014;4(3):509-16.

42. Geda YE, Roberts RO, Mielke MM, Knopman DS, Christianson TJ, Pankratz VS, et al. Baseline neuropsychiatric symptoms and the risk of incident mild cognitive impairment: a population-based study. Am J Psychiatry. 2014;171(5):572-81.

43. Rosenberg PB, Mielke MM, Appleby BS, Oh ES, Geda YE, Lyketsos CG. The association of neuropsychiatric symptoms in MCI with incident dementia and Alzheimer disease. Am J Geriatr Psychiatry. 2013;21(7):685-95.

44. Pietrzak RH, Maruff P, Woodward M, Fredrickson J, Fredrickson A, Krystal $\mathrm{JH}$, et al. Mild worry symptoms predict decline in learning and memory in healthy older adults: a 2-year prospective cohort study. Am J Geriatr Psychiatry. 2012;20(3):266-75.

45. Chung JK, Plitman E, Nakajima S, Chow TW, Chakravarty MM, Caravaggio F, et al. Lifetime History of Depression Predicts Increased Amyloid-beta Accumulation in Patients with Mild Cognitive Impairment. J Alzheimers Dis. 2015;45(3):907-19.

46. Lavretsky H, Siddarth P, Kepe V, Ercoli LM, Miller KJ, Burggren AC, et al. Depression and anxiety symptoms are associated with cerebral FDDNPPET binding in middle-aged and older nondemented adults. Am J Geriatr Psychiatry. 2009;17(6):493-502.

47. Sacuiu S, Insel PS, Mueller S, Tosun D, Mattsson N, Jack CR, Jr., et al. Chronic Depressive Symptomatology in Mild Cognitive Impairment Is Associated with Frontal Atrophy Rate which Hastens Conversion to Alzheimer Dementia. Am J Geriatr Psychiatry. 2016;24(2):126-35.

48. Wilson RS, Boyle PA, Capuano AW, Shah RC, Hoganson GM, Nag S, et al. Late-life depression is not associated with dementia-related pathology. Neuropsychology. 2016;30(2):135-42.

49. Donovan NJ, Okereke OI, Vannini P, Amariglio RE, Rentz DM, Marshall GA, et al. Association of Higher Cortical Amyloid Burden With Loneliness in Cognitively Normal Older Adults. JAMA Psychiatry. 2016;73(12):1230-7.

50. Rosenberg PB. Loneliness as a Marker of Brain Amyloid Burden and Preclinical Alzheimer Disease. JAMA Psychiatry. 2016;73(12):1237-8.

51. Saunders KT, Langbaum JB, Holt CJ, Chen W, High N, Langlois C, et al. Arizona Alzheimer's Registry: Strategy and Outcomes of a Statewide Research Recruitment Registry. J Prev Alzheimers Dis. 2014;1(2):74-9. 